Series in Real Analysis - Volume 9

\title{
THEORIES OF INTEGRATION
}

The Integrals of Riemann, Lebesgue, Henstock-Kurzweil, and Mcshane 


\section{SERIES IN REAL ANALYSIS}

$\forall$ ol. 1: Lectures on the Theory of Integration

D Henstock

$\$$ ol. 2: Lanzhou Lectures on Henstock Integration

Lee Peng Yee

ol. 3: The Theory of the Denjoy Integral \& Some Applications

V G Celidze \& A G Dzvarseisvili

translated by $P S$ Bullen

$\Phi$ ol. 4: Linear Functional Analysis

W Orlicz

ol. 5: Generalized ODE

S Schwabik

Jol. 6: Uniqueness \& Nonuniqueness Criteria in ODE RP Agarwal \& V Lakshmikantham

Vol. 7: Henstock-Kurzweil Integration: Its Relation to Topological Vector Spaces Jaroslav Kurzweil

Vol. 8: Integration between the Lebesgue Integral and the Henstock-Kurzweil Integral: Its Relation to Local Convex Vector Spaces Jaroslav Kurzweil 
Series in Real Analysis - Volume 9

\section{THEORIES OF INTEGRATION}

The Integrals of Riemann, Lebesgue, Henstock-Kurzweil, and Mcshane

\section{Douglas S Kurtz Charles W Swartz}

New Mexico State University, USA 
Published by

World Scientific Publishing Co. Pte. Ltd.

5 Toh Tuck Link, Singapore 596224

USA office: Suite 202, 1060 Main Street, River Edge, NJ 07661

UK office: 57 Shelton Street, Covent Garden, London WC2H 9HE

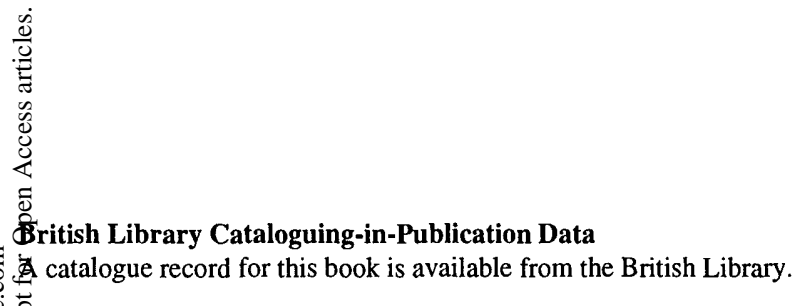

A catalogue record for this book is available from the British Library.

Series in Real Analysis - Vol. 9

THEORIES OF INTEGRATION

he Integrals of Riemann, Lebesgue, Henstock-Kurzweil, and McShane

opyright (C) 2004 by World Scientific Publishing Co. Pte. Ltd.

ll rights reserved. This book, or parts thereof, may not be reproduced in any form or by any means, lectronic or mechanical, including photocopying, recording or any information storage and retrieval ystem now known or to be invented, without written permission from the Publisher.

昰

For photocopying of material in this volume, please pay a copying fee through the Copyright Elearance Center, Inc., 222 Rosewood Drive, Danvers, MA 01923, USA. In this case permission to thotocopy is not required from the publisher.

ใิSBN $981-238-843-5$ 
To Jessica and Nita, for supporting us during the long haul to bring this book to fruition. 
This page intentionally left blank 


\section{Preface}

This book introduces the reader to a broad collection of integration theories, focusing on the Riemann, Lebesgue, Henstock-Kurzweil and McShane integrals. By studying classical problems in integration theory (such as convergence theorems and integration of derivatives), we will follow a historical development to show how new theories of integration were developed to solve problems that earlier integration theories could not handle. Several of the integrals receive detailed developments; others are given a less complete discussion in the book, while problems and references directing the reader to future study are included.

The chapters of this book are written so that they may be read independently, except for the sections which compare the various integrals. This means that individual chapters of the book could be used to cover topics in integration theory in introductory real analysis courses. There should be sufficient exercises in each chapter to serve as a text.

We begin the book with the problem of defining and computing the area of a region in the plane including the computation of the area of the region interior to a circle. This leads to a discussion of the approximating sums that will be used throughout the book.

The real content of the book begins with a chapter on the Riemann integral. We give the definition of the Riemann integral and develop its basic properties, including linearity, positivity and the Cauchy criterion. After spresenting Darboux's definition of the integral and proving necessary and sufficient conditions for Darboux integrability, we show the equivalence of the Riemann and Darboux definitions. We then discuss lattice properties and the Fundamental Theorem of Calculus. We present necessary and sufficient conditions for Riemann integrability in terms of sets with Lebesgue measure 0 . We conclude the chapter with a discussion of improper integrals. 
We motivate the development of the Lebesgue and Henstock-Kurzweil integrals in the next two chapters by pointing out deficiencies in the Riemann integral, which these integrals address. Convergence theorems are used to motivate the Lebesgue integral and the Fundamental Theorem of Calculus to motivate the Henstock-Kurzweil integral.

We begin the discussion of the Lebesgue integral by establishing the sttandard convergence theorem for the Riemann integral concerning uniformly convergent sequences. We then give an example that points out the failure of the Bounded Convergence Theorem for the Riemann integral, and use this to motivate Lebesgue's descriptive definition of the Lebesgue inte要吕. We show how Lebesgue's descriptive definition leads in a natural way to the definitions of Lebesgue measure and the Lebesgue integral. Following fiscussion of Lebesgue measurable functions and the Lebesgue integral, we develop the basic properties of the Lebesgue integral, including convergence theorems (Bounded, Monotone, and Dominated). Next, we compare he Riemann and Lebesgue integrals. We extend the Lebesgue integral to $\frac{0}{2}$-dimensional Euclidean space, give a characterization of the Lebesgue into Theorem on the equality of multiple and iterated integrals. A discussion of the space of integrable functions concludes with the Riesz-Fischer Theorem.

In the following chapter, we discuss versions of the Fundamental Theof rem of Calculus for both the Riemann and Lebesgue integrals and give examples showing that the most general form of the Fundamental Theorem Calculus does not hold for either integral. We then use the Fundamental Theorem to motivate the definition of the Henstock-Kurzweil integral, also now as the gauge integral and the generalized Riemann integral. We deYrelop basic properties of the Henstock-Kurzweil integral, the Fundamental Theorem of Calculus in full generality, and the Monotone and Dominated Eonvergence Theorems. We show that there are no improper integrals in the Henstock-Kurzweil theory. After comparing the Henstock-Kurzweil Integral with the Lebesgue integral, we conclude the chapter with a discusȘion of the space of Henstock-Kurzweil integrable functions and Henstockگं.

Finally, we discuss the "gauge-type" integral of McShane, obtained by slightly varying the definition of the Henstock-Kurzweil integral. We establish the basic properties of the McShane integral and discuss absolute integrability. We then show that the McShane integral is equivalent to the Lebesgue integral and that a function is McShane integrable if and only if it is absolutely Henstock-Kurzweil integrable. Consequently, the McShane 
integral could be used to give a presentation of the Lebesgue integral which does not require the development of measure theory. 
This page intentionally left blank 


\section{Contents}

Preface vii

Introduction 1

1.1 Areas . . . . . . . . . . . . . . . . 1

1.2 Exercises ........................... 9

Riemann integral 11

2.1 Riemann's definition . . . . . . . . . . . . . . . . 11

2.2 Basic properties . . . . . . . . . . . . . . . . 15

2.3 Cauchy criterion . . . . . . . . . . . . 18

2.4 Darboux's definition . . . . . . . . . . . . . . . 20

2.4.1 Necessary and sufficient conditions for Darboux integrability . . . . . . . . . . . . . . 24

2.4.2 Equivalence of the Riemann and Darboux definitions 25

2.4 .3 Lattice properties . . . . . . . . . . . . . . . 27

2.4 .4 Integrable functions . . . . . . . . . . . . 30

2.4.5 Additivity of the integral over intervals . . . . . . 31

2.5 Fundamental Theorem of Calculus . . . . . . . . . . . 33

2.5.1 Integration by parts and substitution . . . . . . 37

2.6 Characterizations of integrability . . . . . . . . . 38

2.6 .1 Lebesgue measure zero . . . . . . . . . . . . . 41

2.7 Improper integrals . . . . . . . . . . . . . . . . . . 42

2.8 Exercises . . . . . . . . . . . . . . . . 46

3. Convergence theorems and the Lebesgue integral 53

3.1 Lebesgue's descriptive definition of the integral . . . . . . 56 
3.2 Measure . . . . . . . . . . . . . . . . . . . 60

3.2 .1 Outer measure . . . . . . . . . . . 60

3.2 .2 Lebesgue Measure . . . . . . . . . . . . . . . . . 64

3.2.3 The Cantor set . . . . . . . . . . . . . 78

3.3 Lebesgue measure in $\mathbb{R}^{n} \ldots \ldots \ldots$. . . . . . . . . . . . . . . . . . . . . . . . . . .

3.4 Measurable functions . . . . . . . . . . . . . . . 85

3.5 Lebesgue integral . . . . . . . . . . . . . . . 96

3.6 Riemann and Lebesgue integrals . . . . . . . . . . . . . 111

3.7 Mikusinski's characterization of the Lebesgue integral . . . 113

3.8 Fubini's Theorem . . . . . . . . . . . . . . . . . . . . 117

3.9 The space of Lebesgue integrable functions . . . . . . . . . 122

3.10 Exercises . . . . . . . . . . . . . . 125

Fundamental Theorem of Calculus and the HenstockKurzweil integral

4.1 Denjoy and Perron integrals . . . . . . . . . . . . . . 135

4.2 A General Fundamental Theorem of Calculus . . . . . . . . 137

4.3 Basic properties . . . . . . . . . . . . . . . . 145

4.3.1 Cauchy Criterion . . . . . . . . . . . . . 150

4.3.2 The integral as a set function . . . . . . . . . 151

4.4 Unbounded intervals . . . . . . . . . . . . . . . 154

4.5 Henstock's Lemma . . . . . . . . . . . . . . . . 162

4.6 Absolute integrability . . . . . . . . . . . . . . . 172

4.6 .1 Bounded variation . . . . . . . . . . 172

4.6.2 Absolute integrability and indefinite integrals . . . 175

4.6 .3 Lattice Properties . . . . . . . . . . . . . . . 178

4.7 Convergence theorems . . . . . . . . . . . . . . . 180

4.8 Henstock-Kurzweil and Lebesgue integrals . . . . . . . . . . 189

4.9 Differentiating indefinite integrals . . . . . . . . . . . . 190

4.9.1 Functions with integral 0 . . . . . . . . 195

4.10 Characterizations of indefinite integrals . . . . . . . 195

4.10.1 Derivatives of monotone functions . . . . . . . . 198

4.10 .2 Indefinite Lebesgue integrals . . . . . . . . . . . . . 203

4.10.3 Indefinite Riemann integrals . . . . . . . . . . . 204

4.11 The space of Henstock-Kurzweil integrable functions . . . . 205

4.12 Henstock-Kurzweil integrals on $\mathbb{R}^{n} \ldots \ldots$. . . . . . 206

4.13 Exercises . . . . . . . . . . . . . . . . . . . . 214

5. Absolute integrability and the McShane integral 223 
5.1 Definitions . . . . . . . . . . . . . . . . . 224

5.2 Basic properties . . . . . . . . . . . . . . . . . . . . . . . . . . . . . . . . . . .

5.3 Absolute integrability . . . . . . . . . . . . . . . . . 229

5.3.1 Fundamental Theorem of Calculus . . . . . . . . 232

5.4 Convergence theorems . . . . . . . . . . . . . . . 234

5.5 The McShane integral as a set function . . . . . . . . . 240

5.6 The space of McShane integrable functions . . . . . . . . 244

5.7 McShane, Henstock-Kurzweil and Lebesgue integrals . . . 245

5.8 McShane integrals on $\mathbb{R}^{n} \ldots \ldots \ldots \ldots . \ldots 253$

5.9 Fubini and Tonelli Theorems . . . . . . . . . . . . 254

5.10 McShane, Henstock-Kurzweil and Lebesgue integrals in $\mathbb{R}^{n} \quad 257$

5.11 Exercises . . . . . . . . . . . . . . . 258

Bibliography 263 